\section{$\underset{\substack{\text { hommes } \\ \text { \& migrations }}}{ }$}

\section{Hommes \& migrations}

Revue française de référence sur les dynamiques

migratoires

$1295 \mid 2012$

Algérie - France : une communauté de destin

\title{
La littérature beur et ses suites
}

Une littérature qui a pris des ailes

\section{Laura Reeck}

\section{(2) OpenEdition}

1 Journals

\section{Édition électronique}

URL : http://journals.openedition.org/hommesmigrations/1077

DOI : 10.4000/hommesmigrations. 1077

ISSN : 2262-3353

Éditeur

Musée national de l'histoire de l'immigration

\section{Édition imprimée}

Date de publication : 1 janvier 2012

Pagination : 120-129

ISSN : 1142-852X

\section{Référence électronique}

Laura Reeck, «La littérature beur et ses suites », Hommes \& migrations [En ligne], 1295 | 2012, mis en

ligne le 01 janvier 2014, consulté le 23 avril 2019. URL : http://journals.openedition.org/

hommesmigrations/1077 ; DOI : 10.4000/hommesmigrations.1077 


\section{La littérature beur et ses suites Une littérature qui a pris des ailes}

Par Laura Reeck, professeure associée à Allegheny College, spécialiste en littérature francophone et en études postcoloniales

L'anniversaire de l'indépendance algérienne offre l'occasion de s'attarder sur les générations qui, en littérature, ont hérité de cette histoire : les générations nées de l'immigration algérienne, coloniale d'abord, postcoloniale ensuite. Ce que l'on a appelé la "littérature beur" est devenu depuis littérature de banlieue, littérature urbaine, populaire, "post-beur". Si certains écrivains rencontrent le succès, ils peinent à se dégager des catégories identitaires et sociales qui tendent à masquer leur talent littéraire. 
Les débuts de la "littérature beur" ${ }^{\text {"(1) }}$ restent marqués par la figure de Mehdi Charef. Né en Algérie en 1952, arrivé en France en 1962, son Thé au harem d'Archi Ahmed (Mercure de France) a sonné l'entrée en scène de la littérature beur en 1983. Le roman explore tous les registres de la langue française, parcourt l'espace des cités et place en son centre le personnage d'un fils d'immigrés algériens, agité, curieux, se cherchant, aimant, isolé au sein même de la foule. Le livre a fait l'effet d'un coup de tonnerre. Charef a été invité sur le plateau de l'émission Apostrophes par Bernard Pivot ; le roman a été adapté au cinéma et a connu un grand succès. Dans le sillage de cette réception enthousiaste, Charef a été consacré comme le premier écrivain beur. Dans les colonnes du Nouvel Observateur, l'écrivain pied-noir Jules Roy a écrit : "Le style de ce bouquin brûle la conscience, ça claque, ça chiale comme dans les romans de Chester Himes où Harlem déborde. Ici, le patrimoine de notre langue s'enrichit, c'est notre littérature que ces bâtards qui auraient dû se contenter de fabriquer nos bagnoles et de balayer nos rues se permettent de peloter et de violer dans des recoins malsains. Tout comme

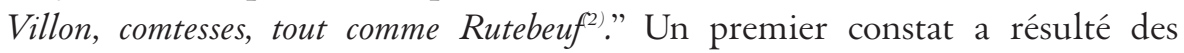
commentaires de Roy : quoique rebelle, Charef pouvait revendiquer des précédents bel et bien français, Rutebeuf et François Villon, agitateurs en leur temps devenus écrivains canoniques du nôtre.

Quant au statut de cette nouvelle littérature, Charef a affirmé n'écrire ni de littérature algérienne, ni de littérature française, ni de littérature beur ${ }^{(3)}$. Il a placé son écriture sous l'égide de la "littérature immigrée", concept nouveau et déroutant : une littérature de nulle part et sans case de départ. Pourtant, dans un entretien donné en 2002, Charef a consolidé le corpus beur avec quelques thèmes, tout en affirmant que la littérature beur se devait de mûrir : "Tant que dans le roman il est toujours question de racines, d'identité, d'intégration c'est comme s'il y avait toujours un pas à faire. Il faut sortir de là. Je pense que c'est pour ça que jai essayé de faire des films où il n'était pas question d'immigration. Mais je pense qu'on est encore au stade où l'on a envie de revoir là d'où l'on vient, de revoir notre enfance et l'identité des parents pour nous en sortir. Une fois qu'on aura fait ce pas analytique, je pense qu'on pourra voler un peu plus. On peut voler un peu... mais on a beaucoup de choses sur les épaules, sur les ailes ${ }^{(4)}$."

Charef a particulièrement et singulièrement aidé à mettre l'Algérie et la France en vis-à-vis historique dans son roman Le Harki de Meriem (Mercure de France, 1989) et sa pièce 1962, qui date de 2005. Quant aux Algériens et aux Français, Charef explique et questionne: "Ils ont non seulement manqué leur rencontre, mais ils ont surtout manqué leur séparation. J'ai toujours voulu savoir pourquoi il n'y a pas eu d'échange, juste une coexistence, quand les Français étaient en Algérie. Pourquoi aussi les Français sont partis dans un silence terrible ${ }^{(5)}$." Scruter ce silence à travers la parole littéraire, comme continue à le faire Charef, était sans doute l'une des étapes à franchir. 


\section{Le droit à l'indifférence}

Si la Marche pour l'égalité et contre le racisme de 1983 fit couler beaucoup d'encre dans la presse, elle donna également naissance à une forte et énergique vague d'ceuvres de fiction et de témoignages. Trois ans plus tard, en 1986, sortaient les romans Le Gone du Chaâba (Seuil) d'Azouz Begag, Georgette! (Barrault) de Farida Belghoul et Le Sourire de Brahim (Denoël) de Nacer Kettane. Cette première vague charriait les thèmes de la famille, de l'école et de la rue...L'horizon des personnages, encore pénétrés de "nostalgérie", dépendait en partie d'une attente d'un retour au pays natal, dans cette Algérie qui, déjà, n'avait plus rien de "natal" pour la plupart d'entre eux. Le ressac libéra, en un fracas assourdissant, un profond questionnement sur l'identité portée par des personnages interprètes-traducteurs servant de passerelles entre les parents et la société française. Ces écrivains de la première vague ont dû alors ferrailler contre les discours ambiants sur l'intégration et les préjugés du moment. Comme ses consceurs, Soraya Nini (Ils disent que je suis une beurette, Fixot, 1993) et Sakinna Boukhedanna (Journal. "Nationalité : immigré(e),, L'Harmattan, 1987), Farida Belghoul n'a écrit qu'un seul texte, Georgette!, un roman-psychologique-choc dans lequel une petite fille nous entretient, sous la forme d'un monologue intérieur, de son apprentissage de l'écriture à l'école. Comme on lui prédisait une longue et prometteuse carrière littéraire, le silence de Belghoul depuis ce premier et unique roman continue de résonner très fort. Au moment même où elle rédigeait son roman, Belghoul occupa le devant de la scène politique et s'adressa aux manifestants de la Marche de 1983 et à ceux de Convergence 1984. À cette occasion, elle a été amenée à se positionner dans sa Lettre ouverte aux gens convaincus: "Le droit à la différence est une concession du dominé. L'enjeu n'est pas la reconnaissance des différences qui de toute façon existent, mais plutôt la conquête des droits universels." En cela, Belghoul s'est démarquée du communautarisme, et donc d'une frange du mouvement "beur". Quoique ce discours, qui s'attache vraisemblablement au modèle républicain, puisse paraître un discours peu révolutionnaire, il a été stratégique. Il revenait à dire que nous avons tous le droit aux droits universels. Faute de vision politique collective, le mouvement beur a stagné dès 1984, et Belghoul s'est retirée de la vie publique. Pour autant, elle n'a jamais arrêté de se battre. Sauve qui peut de la documentariste algérienne Samia Chala (2007) montre la lutte de Belghoul contre l'illettrisme, via la création de l'association REID qui propose une "école familiale" à la maison pour remettre à niveau des élèves en situation d'échec scolaire. Ainsi, Belghoul poursuit son action, encore et toujours en faveur de l'écriture.

Nacer Kettane n'a lui aussi écrit qu'un seul roman, Le Sourire de Brahim, qui décrit, globalement, le paysage socio-associatif entourant la Marche. Le souci principal chez 
Kettane semble avoir été l'expression de la nouvelle identité "beur". Sur ce point, il ne faut pas oublier qu'il a cofondé en 1981 la chaîne Radio Beur, devenue aujourd'hui Beur FM, dont il est le PDG, et a été directeur général de Beur TV de 2003 à 2011. En 1986, il publie un essai, Droit de réponse à la démocratie française (La Découverte), dans lequel il esquisse le portrait d'une France en devenir grâce au métissage algéro-français : "Mutants déchirés de la 'Mc Donald's-couscous-steak frites society', nous sommes là, que vous le vouliez ou pas. Réalité incontournable, car chacun d'entre nous est différent de l'autre et interpelle à sa façon le passé, le présent et l'avenir ${ }^{(6) . "}$ Ici, il pose l'universalité de la différence. Autrement dit, les identités identiques n'existent pas, ou comme Amin Maalouf l'a dit : "Mon identité, c'est ce qui fait que je ne suis identique à aucune autre personne ${ }^{(7)}$." Dans cette perspective, les données changent : il devient possible de cumuler, de multiplier les références à sa guise. Ou, comme le romancier-chanteur Mohand Mounsi le formule dans Territoire d'outreville (Stock, 1995), de rejeter à la fois l'intégrisme et l'intégration : "Je revendique l'intégrité, c'est-à-dire être ni ceci ni cela mais toute chose ${ }^{(8) "}$.

Ces écrivains ont semé les graines d'un nouveau paysage identitaire. Pourtant, cette semence n'a donné aucun fruit au niveau officiel, où les débats se sont limités aux questions de la double nationalité, de la volonté citoyenne, de l'affaire du foulard... sans jamais aller plus loin.

\section{De l'instrumentalisation d'un écrivain beur}

Avec sa vaste collection d'essais sur la mobilité sociale et physique dans les banlieues défavorisées, en passant par son poste au gouvernement de Dominique de Villepin comme ministre délégué à la Promotion de l'Égalité des chances (voir son essai sur la discrimination au sein du gouvernement, Un mouton dans la baignoire, Fayard, 2007), Azouz Begag se distingue par sa volonté inébranlable de relier ceux qu'il appelle "les Franco-Français" et "les Français issus de l'immigration visible". Begag a été vivement critiqué par certains pour le poste ministériel qu'il occupait - rassemblant contre lui aussi bien les partisans de la gauche que les fidèles d'Oumma.com. Pourtant, il ne faut pas sous-estimer son travail sur les corrélations entre les lieux de résidence et l'enseignement, le travail, voire une certaine vision du monde. Ainsi, dans son rapport La République à ciel ouvert (La Documentation française, 2004), qui insiste sur la distance relationnelle entre la police et les jeunes vivant dans les banlieues défavorisées, il a prédit, d'une certaine façon, les émeutes de 2005. Dans ce rapport, Begag propose mesures et moyens concrets pour que la police nationale française reflète davantage la population, via notamment une politique de 
recrutement et de formation qui intègre "les minorités visibles". De façon paradoxale, quand les émeutes ont éclaté et alors que Begag siégeait au gouvernement comme ministre, le Premier ministre, Dominique Villepin, a trouvé bon de lui demander de rester dans son bureau au lieu de se rendre sur le terrain où il a grandi et sur lequel il travaille et réfléchit comme chercheur depuis une trentaine d'années. C'est à ce moment-là que Begag a compris qu'il était devenu "le Beur de service”. Il a appris que ses collègues du gouvernement, notamment l'actuel président de la République Nicolas Sarkozy, voulaient qu'il reste à sa place. Il a démissionné en avril 2007, près de deux ans plus tard.

En 2009, après une douzaine de romans et une vingtaine de récits de jeunesse, Azouz Begag a publié un nouveau livre pour enfants, Dites-moi Bonjour! (Fayard), où il est question d'un gamin qui se fait guider par "sa bonne étoile" vers un autre univers. Lorsque l'enfant doit retourner sur terre, il découvre le nouvel ordre suprasécuritaire d'une pieuvre géante. Le pire, selon la quatrième de couverture : "Elle gave les citoyens pour les empêcher de penser. Au bout du conte, le narrateur choisit de laisser les citoyens prendre en charge eux-mêmes leur destin. Et, dans son sillage, se répand le goût du combat pour la liberté." Il n'est pas difficile de lire entre les lignes et de voir ici un appel à la liberté de pensée, chose qui aurait été refusée à Begag quand il fréquentait les allées du pouvoir. Aujourd'hui, Begag renoue avec l'écriture et parraine l'ONG Bibliothèques sans frontières, qui cherche à démocratiser l'accès au savoir et à la lecture dans le monde. En 2012, à l'occasion du 50 anniversaire de l'indépendance algérienne, paraîtra une bande dessinée qui, en hommage, reconstruit la vie de ses parents dans le village d'El-Ouricia avant leur émigration vers la France.

\section{Après le temps des revendications, celui des comptes}

Très vite, après 1986, cette première vague d'écrivains d'origine algérienne répertoriés comme écrivains "beurs" a grossi. À l'image de Nina Bouraoui, Tassadit Imache, Mohand Mounsi ou Akli Tadjer, de nombreux écrivains se sont affirmés. S'ils sont nombreux à s'être lancés dans la carrière, seule Nina Bouraoui s'est vue gratifiée d'un prix littéraire de renom, en l'occurrence le prix Renaudot, pour son autofiction Mes mauvaises pensées (Stock, 2005). Mais, malgré son dynamisme et son potentiel, cette littérature reste largement une littérature peu reconnue et souvent même méconnue par le lectorat et les institutions littéraires françaises. Cette question de la réception et de la reconnaissance de ces écrivains demeure épineuse. C'était sans doute en partie pour remédier à ce problème - que les minorités 
visibles en France sont auteurs d'une littérature invisible - que le groupe Qui fait la France ? a publié un manifeste littéraire en $2007^{(9)}$. Le collectif, composé d'écrivains d'origines différentes mais partageant un vécu ou un engagement dans les banlieues défavorisées, s'est formé suite aux émeutes de 2005. Le projet littéraire du collectif va de pair avec une remise en question des référents identitaires de la société française. Plus de vingt ans après la Marche pour l'égalité et contre le racisme, ces écrivains doivent encore insister sur l'égalité et sur leur inclusion dans le champ littéraire français. "Parce que catalogués écrivains de banlieues, étymologiquement le lieu du ban, nous voulons investir le champ culturel, transcender les frontières et ainsi récupérer l'espace confisqué qui nous revient de droit, pour l'aspiration légitime à l'universalisme. Parce que cette génération, la nôtre, a le feu pour réussir, le punch pour démolir les portes, la rage pour arriver au but, le charisme pour crever l'écran, l'intelligence pour rafler les diplômes, la force pour soulever les barricades, la hargne du sportif, la beauté du livre, le caractère de l'Afrique, l'odeur du Maghreb, l'amour du drapeau tricolore et de la poésie de France." Le lexique rappelle

\section{Par leur intervention, les membres de Qui fait la France? appellent à l'action,} dénoncent les privilèges hérités de père en fils, et s'insèrent dans le paysage littéraire, tout en revendiquant leur droit à un universalisme supposé être bienveillant. celui de Nacer Kettane dans Droit de réponse à la démocratie française ou celui de Mohand Mounsi dans Territoire d'outre-ville féroce, décidé, angoissé, énergique. Mais les membres du collectif sont plus colériques. Ainsi, selon Mohamed Razane, le président du collectif, "on considère qu'effectivement la Marche des Beurs, qui va bientôt dater de trente ans, a été un soulèvement qui a interpellé les gens sur les réalités sociales. Ils [les Beurs] ont usé de toutes les ficelles qu'on pourrait inclure dans la notion du débat. La situation d'aujourd'hui s'est dégradée ... on n'est pas là pour discuter, on est là pour demander des comptes, et nous fâcher ${ }^{(10)}$." Par leur intervention, les membres de Qui fait la France? appellent à l'action, dénoncent les privilèges hérités de père en fils, et s'insèrent dans le paysage littéraire, tout en revendiquant leur droit à un universalisme supposé bienveillant.

Deux écrivains du collectif, Karim Amellal et Faïza Guène, retiennent notre attention. Le premier, diplômé de l'Institut d'études politiques de Paris, enseignant à Sciences Po, bloggeur en plus d'être auteur de romans et d'essais, a créé en 2010 sa propre entreprise médiatique, Stand Alone Media. L'une des priorités du collectif est de s'attaquer aux images propagées dans les médias de ceux qu'Amellal appelle "les minorités ethniques" - nouveau mot du lexique français et nouvelle conception pour 
les penseurs de l'identité. Son essai Discriminez-moi (Flammarion, 2005) s'avère un plaidoyer pour la discrimination positive. Son mot d'ordre est : "Reconnaissons les origines culturelles, les minorités ethniques, les différences, sans cesser d'être français ${ }^{(11)}$." De surcroît, il en appelle à régler le problème d'une société qui fonctionne à "deux vitesses $^{(12)}$ ", rejoignant en cela Azouz Begag et sa comparaison-impératif dans La République à ciel ouvert visant à corriger le différentiel qui permet à certains de rouler plus vite que d'autres. Amellal et Begag auraient peut-être des choses à partager, surtout depuis que ce dernier a quitté le gouvernement ${ }^{(13)}$.

\section{Reconnaissance internationale contre invisibilité nationale}

En 2004, Kiffe kiffe demain de Faïza Guène fit beaucoup de vagues en France, mais aussi dans le monde, puisque ce premier roman a été traduit en une douzaine de langues. La presse anglo-saxonne a comparé Guène à Zadie Smith ou à Monica Ali et a souligné ses nombreuses références à la culture américaine. Mais là où cette presse situe Guène dans un contexte et des référents littéraires et culturels, la presse française fait d'elle plus souvent un sujet des pages "société" qu'un écrivain mentionné dans les pages "culture" ou "littérature". Une différence de traitement qui révèle un problème de réception bien enraciné. Cette réception en France est peut-être encore plus compliquée du fait que Faïza Guène semble incarner, à l'instar de Farida Belghoul, la révolte, une révolte totale qui peut mener à tout, même au silence - et surtout au silence lorsque l'on est sommé de parler. Depuis qu'elle occupe le devant de la scène littéraire, Guène refuse d'être "l'invitée de service" aux réceptions culturelles du gouvernement ${ }^{(14)}$. Elle dit ce qu'elle a à dire dans ses romans et ses courts métrages de plus en plus nombreux, dans lesquels elle présente la vie en banlieue du point de vue de ceux qui y vivent. Comme beaucoup de ses aînés, Guène refuse la perspective de surplomb.

Concernant la question des prix littéraires, Faïza Guène pointe le problème, "The big prizes? Are you crazy? Never, never in my life will I get a prize. That would mean recognising that what I write is literature, that there are intellectuals in the banlieues ${ }^{(15)}$."Cela témoigne de l'écart que vivent certains de ces écrivains accueillis chaleureusement à l'étranger mais avec toujours beaucoup de retenue en France. Les traductions en plusieurs langues des ceuvres de Faïza Guène et d'Azouz Begag ; le film collectif et international sur l'enfance, Les Enfants invisibles (2005), qui réunit plusieurs réalisateurs, dont Mehdi Charef (aux côtés d'Emir Kusturica, Spike Lee, Katia Lund, Jordan Scott, Ridley Scott, Stefano Veneruso, John Woo) ; le parrainage de l'ONG 
Bibliothèques sans frontières par Azouz Begag ; le fait que Karim Amellal écrit des articles pour des revues étrangères ou que Rachid Djaïdani a été sélectionné par la ville de New York dans le cadre d'une bourse Stendhal... sont autant de signes de cette notoriété et reconnaissance internationales pour ces écrivains. À cet égard, il faut signaler le message de Rachid Djaïdani à la fin de son documentaire autofilmé, Sur ma ligne (2006), et ce qui l'a poussé à réaliser ce film : l'accusation portée contre lui par les éditions du Seuil, prétendant qu'il n'était pas l'auteur de son premier roman, Boumkceur (1999). Djaïdani a donc décidé de filmer le "making-of" de son deuxième roman, Mon nerf(Seuil, 2004). Avec ce documentaire, il administre la preuve que "je est un auteur" et qu'il entend être lu et approuvé comme tel.

\section{D'une identité-monde à un impossible chez-soi}

Cette tension dans la réception de ces écrivains est frappante, le jeu entre visibilité et invisibilité persiste. Une ouverture pour les écrivains d'origine algérienne se dessine peut-être dans la perspective du projet pour une littérature-monde. Le manifeste Pour une littérature-monde en français de Michel Le Bris et Jean Rouaud est paru dans Le Monde des livres quelques mois avant que le manifeste de Qui fait la France? ne sorte dans Le Nouvel Observateur et dans Les Inrockuptibles. Parmi les 44 signataires de ce manifeste qui prononce l'acte de décès de la francophonie à la faveur d'une littérature-monde en français ne distinguant plus le centre de la périphérie, ne figure aucun écrivain ayant des attaches algériennes. Le Bris et Rouaud ont corrigé cette absence dans leur deuxième ceuvre collective, Je est un autre. Pour une identité-monde (Gallimard, 2010). Azouz Begag, Ahmed Kalouaz et Leila Sebbar y ont chacun écrit un texte. En introduction, Michel Le Bris et Jean Rouaud expliquent que la collection se veut une réponse au débat sur l'identité française lancé en 2009 par le gouvernement français. Ils tenaient à inviter des écrivains pour critiquer, par la voix poétique, cette initiative. Azouz Begag insiste sur le contexte global dans lequel il écrit, mettant en scène une rencontre avec un Africain à Los Angeles et la sympathie qu'il témoigne à tous les citoyens-nomades. Leïla Sebbar souligne son double héritage, "que je peux signer du nom de mon père dans la langue de ma mère" ${ }^{(16) " .}$

Ahmed Kalouaz offre l'intervention la plus inattendue. Sa contribution, Ça me revient tout ça, est remarquable, d'abord et avant tout parce qu'elle ne vise pas le monde, le global. Ensuite, non sans subtilité et force, le texte traite, de façon oblique, de l'injustice et des plaies historiques algéro-françaises qui saignent encore. En intercalant deux chansons, La grille de Jean-Max Brua, chanson anti-capitaliste, et $Y a$ 
Rayah de Dahmane El Harrachi, chanson chaâbi sur l'émigration, l'exil, les déplacés, Ahmed Kalouaz lie le destin de la génération immigrée des pères à la machine capitaliste et au broyage des âmes. C'est donc un texte hommage que l'auteur d'Avec tes mains (Les éditions du Rouergue, 2009) offre à ces hommes qui ont quitté leur terre natale sans savoir ce qui les attendait à l'autre bout de l'horizon. Cette génération en a enfanté une autre : "Vous partez seuls, mais vous préparez à ceux qui y viendront après la traversée un avenir curieux. Nés là-bas, on vous offre si peu de droits, nés ici, il faudra qu'ils prouvent chaque jour que la terre qu'ils foulent est bien la leur ${ }^{(17) . " ~ N i ~ l e s ~ p e ̀ r e s ~ n ' o n t ~ e ́ t e ́, ~ n i ~ l e s ~ e n f a n t s ~ n e ~ s o n t, ~ a ̀ ~ p r o p r e m e n t ~ p a r l e r, ~ c h e z ~ e u x . ~ I c i ~}$ Kalouaz met en rapport l'époque coloniale en Algérie et l'époque post-coloniale en France. Il va plus loin en évoquant la manifestation du 17 octobre 1961 à Paris où, selon les sources, de 30 à plus de 300 Algériens ont trouvé la mort sous les coups de la police ou noyés dans la Seine ${ }^{(18)}$. Il se met donc en position de témoigner, de se souvenir des morts, ceux tombés au champ d'honneur du travail et ceux engloutis par les flots, de la mer Méditerranée ou de la Seine. Sa représentation de l'identitémonde s'arrête là, autrement dit elle n'y figure pas. C'est là l'originalité de la contribution de Kalouaz : si sa participation au projet de Michel Le Bris et de Jean Rouaud est sans doute valorisante pour Kalouaz en tant qu'écrivain, dans son texte il soulève avec justesse la face cachée de l'identité-monde. Il suggère qu'il ne faut pas oublier le local dans cette époque qui nous invite sans cesse à penser le global. Dans la marche du monde, il fait valoir que le local est souvent lui-même globalisé et que la diversité se trouve chez soi. Face à cette diversité d'expériences, de langues, d'histoires, il existe aussi des devoirs, dont le devoir de mémoire.

\section{Conclusion}

Littérature née d'un déplacement originel, littérature en mouvement, la "littérature beur" née dans les années quatre-vingt et portée notamment par des écrivains français d'origine algérienne, s'est diversifiée pour offrir un corpus littéraire plus large, qui a mûri. Elle a des lecteurs aux quatre coins du monde, et ses écrivains rayonnent, agissent et s'associent à l'échelle internationale. Pourtant, de vives tensions demeurent quant à sa place dans le champ littéraire français, un champ qui pourrait s'effacer pour céder la place au "French Global" ou à la littérature-monde. Tandis que la diversité de la langue française dans le monde est bien réelle, la diversité en France provoque toujours trouble, agitation et ignorance. La lutte pour la reconnaissance n'est pas finie, et des écrivains tels que Karim Amellal, Azouz Begag, Rachid Djaïdani, Faïza Guène ou Ahmed Kalouaz y sont encore engagés, chacun à sa façon. Car la 
diversité habite la diversité. De Lakhdar Belaïd à Mouloud Akkouche, d'Ahmed Kalouaz à Azouz Begag, de Farid Boudjellal à Ramdane Issad, de Tassadit Imache au duo frère-sceur Mabrouk Rachedi-Habiba Mahany..., on peut tout voir et tout lire. La littérature de la post-migration algérienne est aujourd'hui protéiforme : polar en banlieue, littérature de jeunesse, bande dessinée, roman philosophique, roman social, roman à deux, témoignage, autofiction, nouvelle... De par leur nombre et la variété de leurs écrits, les écrivains d'origine algérienne volent, aujourd'hui, de leurs propres ailes. Le væeu de Mehdi Charef est exaucé.

\section{Notes}

1. Ici, j'utilise le terme souvent critiqué de "beur" pour qualifier la première vague de romans des écrivains d'origine algérienne. C'est pour les situer dans ce moment, le "moment beur" des années quatre-vingt, où l'identité beur prenait forme et se discutait. Ensuite, j'utilise le terme "écrivain d'origine algérienne" afin de délimiter le champ littéraire en question pour coöncider avec ce numéro spécial. C'est-à-dire que je ne traiterai ici que des écrivains ayant des attaches algériennes, quoique la littérature beur comprenne également les écrivains d'origine marocaine.

2. Jules Roy, "Ahmed sortit à cinq heures", in Le Nouvel Observateur, n 22, avril 1983, p. 58.

3. Christiane Achour, Anthologie de la littérature algérienne de langue française, Alger et Paris, Bordas Francophonie, 1990 , p. 184.

4. Mehdi Charef, entretien avec Laura Reeck, in Le Maghreb littéraire, $\mathrm{n}^{\circ}$ 9, 2005, p. 102.

5. 1962, le dernier voyage de Mehdi Charef, $\mathrm{n}^{\circ}$ 1187, Paris, L'avant-scène théâtre, 2005, p. 66.

6. Nacer Kettane, Droit de réponse à la démocratie française, Paris, La Découverte, 1986, p. 19.

7. Amin Maalouf, Les Identités meurtrières, Paris, Grasset, 1998, p. 18.

8. Mohand Mounsi, Territoire d'outre-ville, Paris, Stock, 1995, pp. 123-24.

9. Le manifeste se trouve notamment au début du livre collectif du groupe, Chroniques d'une société annoncée (Stock, 2007). Pour en savoir plus sur le moment social et littéraire dans lequel le manifeste s'inscrit, surtout par rapport au manifeste "Pour une littérature-monde" paru six mois plus tôt, voir l'article de David Murphy (pp. 67-86) et mon article (pp. 258-73) dans Alec G. Hargreaves, Charles Forsdick, et David Murphy (dir.), Transnational French Studies. Postcolonialism et littérature-monde, Liverpool, Liverpool University Press, 2010.

10. Entretien non publié (le 13 décembre 2008) de Mohamed Razane par Laura Reeck.

11. Karim Amellal, Discriminez-moi, Paris, Flammarion, 2005, p. 39.

12. Ibid., p. 16 .

13. Même si, à première vue, Begag ne ressemble pas à Amellal dans son choix de camp politique, et surtout dans le sens où le premier n'a jamais prôné la discrimination positive, en lisant entre les lignes du rapport de 2004 de Begag, on trouve une dizaine de mesures que l'on pourrait facilement lier à la discrimination positive.

Il ne me semble pas que les deux aient des perspectives si différentes que cela. Par ailleurs, Amellal témoigne d'un respect, du moins littéraire, pour Begag dans une intervention récente autour de la littérature ("Méritocratie contre discriminations", www.youtube.com/watch? v=ptTLVA06eDY).

14. Angelique Chrisafis, The Guardian. <http://www.guardian.co.uk/books/2008/jun/05/culture.news/print>

15. Ibid.

16. Je est un autre. Pour une identité-monde, Paris, Gallimard, 2010, p. 107.

17. Ibid., p. 174.

18. De nombreux écrivains d'origine algérienne ont traité de cette date charnière de la guerre d'Algérie, c'est-à-dire quand la guerre a débordé sur le sol de la métropole. Voir Nacer Kettane (Le Sourire de Brahim, 1986), Mohand Mounsi (Territoire d'outre-ville, 1995), Leïla Sebbar (La Seine était rouge, 1999), ainsi que le film documentaire d'Agnès Denis et de Mehdi Lallaoui (Le Silence du fleuve, 1999), et le court métrage de ce dernier, À propos d'octobre (2011). 